\title{
Pharmacokinetic and pharmacodynamic profile of bendamustine and its metabolites
}

\author{
Mona Darwish $^{1} \cdot$ Mary Bond $^{2} \cdot$ Edward Hellriegel $^{3} \cdot$ Philmore Robertson Jr. $^{3} \cdot$ \\ James P. Chovan ${ }^{3}$
}

Received: 23 December 2014 / Accepted: 15 March 2015 / Published online: 1 April 2015

(c) The Author(s) 2015. This article is published with open access at Springerlink.com

\begin{abstract}
Purpose Bendamustine is a unique alkylating agent indicated for the treatment of chronic lymphocytic leukemia and rituximab-refractory, indolent B cell non-Hodgkin's lymphoma. Despite the extensive experience with bendamustine, its pharmacokinetic profile has only recently been described. This overview summarizes the pharmacokinetics, pharmacokinetic/pharmacodynamic relationships, and drug-drug interactions of bendamustine in adult and pediatric patients with hematologic malignancies.

Methods A literature search and data on file (including a human mass balance study, pharmacokinetic population analyses in adult and pediatric patients, and modeling analyses) were evaluated for inclusion.

Results Bendamustine concentrations peak at end of intravenous infusion $(\sim 1 \mathrm{~h})$. Subsequent elimination is triphasic, with the intermediate $t_{1 / 2}(\sim 40 \mathrm{~min})$ as the effective $t_{1 / 2}$ since the final phase represents $<1 \%$ of the area under the curve. Bendamustine is rapidly hydrolyzed to monohydroxy-bendamustine and dihydroxy-bendamustine, which have little or no activity. Cytochrome P450 (CYP) 1A2 oxidation yields the active metabolites
\end{abstract}

Mona Darwish formerly with Teva Branded Pharmaceutical Products R\&D, Inc., Frazer, PA, USA.

Mary Bond

Mary.Bond@Tevapharm.com

1 Sci-Med Bridge, LLC, 1916 General Alexander Drive, Malvern, PA 19355, USA

2 Teva Branded Pharmaceutical Products R\&D, Inc., 41 Moores Road, Frazer, PA 19355, USA

3 Global Nonclinical DMPK, Teva Branded Pharmaceutical Products R\&D, Inc., 145 Brandywine Parkway, West Chester, PA 19380, USA $\gamma$-hydroxybendamustine and $N$-desmethyl-bendamustine, at low concentrations, which contribute minimally to cytotoxicity. Minor involvement of CYP1A2 in bendamustine elimination suggests a low likelihood of drug-drug interactions with CYP1A2 inhibitors. Systemic exposure to bendamustine $120 \mathrm{mg} / \mathrm{m}^{2}$ is comparable between adult and pediatric patients; age, race, and sex have been shown to have no significant effect on systemic exposure in either population. The effect of hepatic/renal impairment on bendamustine pharmacokinetics remains to be elucidated. Higher bendamustine concentrations may be associated with increased probability of nausea or infection. No clear exposure-efficacy response relationship has been observed. Conclusions Altogether, the findings support dosing based on body surface area for most patient populations.

Keywords Chronic lymphocytic leukemia · Indolent B cell non-Hodgkin's lymphoma - Alkylating agent . Pharmacokinetic profile $\cdot$ Systemic exposure

\section{Introduction}

Bendamustine hydrochloride is a unique multifunctional alkylating agent with demonstrated clinical activity against various hematologic malignancies when used as monotherapy or in combination with other chemotherapeutic agents [1-6]. Administered as an intravenous infusion, bendamustine is currently indicated for the treatment of chronic lymphocytic leukemia (CLL) and indolent B cell non-Hodgkin's lymphoma (NHL) that has progressed during or within 6 months of treatment with rituximab or a rituximab-containing regimen [3, 7-9]. The recommended bendamustine dose is $100 \mathrm{mg} / \mathrm{m}^{2}$ on days 1 and 2 of a 28 -day cycle for CLL and $120 \mathrm{mg} / \mathrm{m}^{2}$ 
Fig. 1 Bendamustine and its main metabolites. Reproduced with permission of ASPET [23]

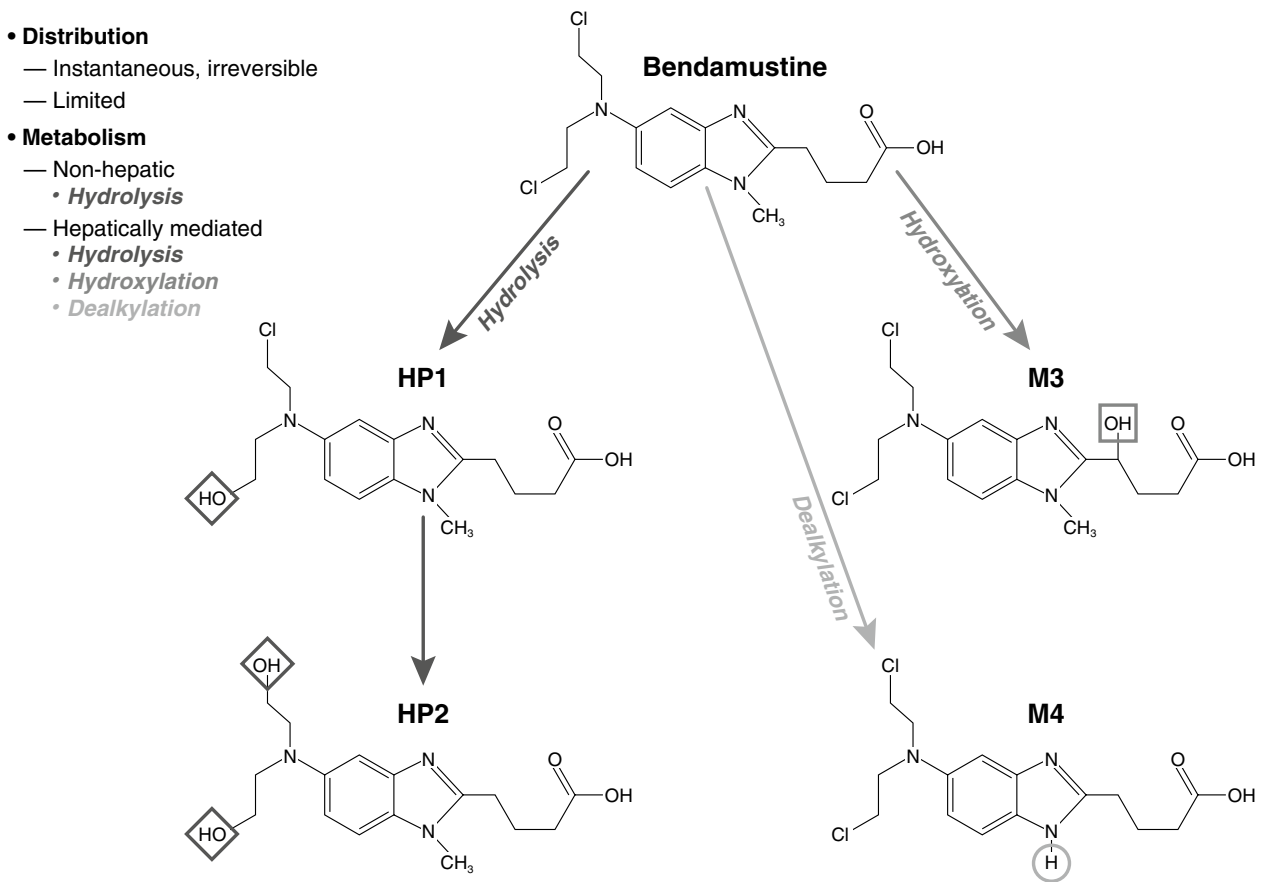

on days 1 and 2 of a 21-day cycle for relapsed/refractory NHL [7].

Although its mechanisms of action have not been fully elucidated, bendamustine, like other bifunctional alkylators, crosslinks DNA and produces single- and doublestrand breaks; however, in vitro studies have demonstrated that bendamustine causes more extensive and durable breaks than carmustine and cyclophosphamide [10]; has incomplete cross-resistance with other alkylators; and leads to cell death via apoptosis or mitotic catastrophe $[1,10]$.

Chemically, bendamustine is 4-[5-[bis(2-chloroethyl) amino]-1-methyl-benzoimidazol-2-yl]butyric acid hydrochloride [11]. Structurally, bendamustine consists of three moieties: a mechlorethamine group with alkylating properties, a butyric acid side chain that increases water solubility, and a benzimidazole ring that may confer an antimetabolite property (Fig. 1) [11-13]. Bendamustine is primarily metabolized via hydrolysis of its mechlorethamine group into two metabolites with little or no activity: monohydroxy-bendamustine (HP1) and dihydroxy-bendamustine (HP2) [7, 14]. Bendamustine also undergoes phase 1 metabolism via cytochrome P450 (CYP) 1A2-catalyzed oxidative pathways, which result in two active circulating metabolites: $\gamma$-hydroxybendamustine (M3), which was previously thought to be $\beta$-hydroxybendamustine $[15,16]$, and $N$-desmethyl-bendamustine (M4) [16]. M3 is produced by $\gamma$-oxidation of the butyric acid side chain, and M4 by demethylation of the benzimidazole ring [16].

Despite the extensive clinical experience with bendamustine, its overall pharmacokinetics, pharmacokinetic/pharmacodynamic relationships, and drug-drug interactions have only recently been described. This report provides a comprehensive overview of data characterizing the pharmacokinetics and pharmacokinetic/pharmacodynamic relationships of bendamustine and its circulating active metabolites in adult and pediatric patients with hematologic malignancies. A MEDLINE search for "bendamustine AND (pharmacokinetics OR pharmacodynamics)" was conducted, and the resulting citation list was reviewed for relevant data to be included in this paper. In addition, data on file from the preclinical and clinical development programs, including modeling analyses, were evaluated for possible relevance and inclusion. Specific datasets included a human mass balance study and population pharmacokinetic analyses in adult and pediatric patients.

\section{Overall pharmacokinetic profile}

As shown by a population pharmacokinetic analysis from a phase 3 study in patients with rituximab-refractory, indolent B cell NHL and a human mass balance study [17, 18], bendamustine is metabolized via multiple pathways, has a short effective $t_{1 / 2}(\sim 40 \mathrm{~min})$ with the maximum plasma concentration $\left(C_{\max }\right)$ typically reached near the end of the infusion period $(\sim 1 \mathrm{~h})$, and a low ratio of concentration at $12 \mathrm{~h}$ to $C_{\max }$ (mean 1:25,000). Thus, although the pharmacokinetics of multiple-dose administration of bendamustine have not been studied, dose accumulation is not expected with the standard dosing schedule of two consecutive days in a 21- or 28-day cycle. Therefore, the single-dose pharmacokinetic profile is considered representative of the multiple-dose pharmacokinetic profile [17, 18]. 


\section{Distribution}

In vitro, approximately $95 \%$ of bendamustine is protein bound (mostly to albumin) [7, 19]. Data suggest that bendamustine is not likely to displace or be displaced by highly protein-bound drugs [19].

In mice administered $\left[{ }^{14} \mathrm{C}\right]$ bendamustine, tissue levels of radioactivity were substantially higher in the liver and kidneys - two highly perfused organs involved in the clearance of bendamustine and its metabolites-than other tissues [20]. In human adults, the mean steady-state volume of distribution for bendamustine was estimated in two studies to be $\sim 25$ L [17] and 20 L [18], which, consistent with murine radioactivity findings, indicates that the drug is mainly confined to the extracellular fluid and not extensively distributed to tissues [7]. This pattern of distribution is also consistent with the effectiveness of bendamustine in inhibiting the growth of lymphoma tumors in xenograft models [21, 22].

\section{Metabolism}

Bendamustine is primarily hydrolyzed (mainly nonenzymatically) to the much less active HP1 and HP2 [7, 14, 23]. Bendamustine is also metabolized by CYP1A2 enzymes to active M3 and M4 [16, 23], which reach their maximum concentrations at or near the same time as the parent drug [17]. The potency of M3 is approximately equivalent to that of bendamustine, although that of M4 is five- to tenfold less than that of the parent drug [16]. Because plasma concentrations of M3 and M4 are only 1/10 and 1/100 that of bendamustine, respectively [17], the contribution of M3 and M4 to the therapeutic activity of bendamustine would be expected to be minimal $[7,16,18]$. In addition, these low plasma concentrations suggest that hepatic metabolism via the CYP1A2 oxidative pathways plays a minor role [23].

Most metabolic pathways for bendamustine were originally observed in rats, such as hydrolysis (the primary metabolic process), cysteine conjugation of the mechlorethamine moiety, and $N$-demethylation and $\gamma$-hydroxylation of the benzimidazole and butyric acid moieties, respectively [24].

Analyses of urine samples from the human mass balance study were conducted to more fully characterize the metabolite profile of bendamustine [23]. Urine samples collected $2 \mathrm{~h}$ after the start of $\left[{ }^{14} \mathrm{C}\right]$ bendamustine infusion were used because they were found to have the highest concentrations of radioactivity. Radiochromatograms of these samples revealed a total of 25 bendamustine-related compounds as well as bendamustine. The majority of the metabolites that were detected were present only during the early periods of sample collection. $\left[{ }^{14} \mathrm{C}\right]$ Bendamustine-derived materials that were detectable in late urine samples (up to $168 \mathrm{~h}$ after the infusion) included products of dihydrolysis and cysteine conjugation of bendamustine and $\gamma$-hydroxybendamustine.

Although a few new metabolic products were detected in the human mass balance study that had not been observed in rats, those products were largely found to represent adducts that formed by reaction of bendamustine with endogenous compounds in the urine, e.g., phosphate, creatinine, and uric acid. These reactions were reproduced in vitro by incubation of bendamustine with urine from a drug-naïve subject.

Overall, these findings indicate that the metabolic elimination of bendamustine is qualitatively the same in humans and rats [23].

\section{Elimination}

The plasma concentration versus time profile of bendamustine declines in a polyphasic manner in the phase 3 population pharmacokinetic study and the human mass balance study, with an effective $t_{1 / 2}$ of $\sim 40 \mathrm{~min}[17,18]$. The active metabolites M3 and M4 have a $t_{1 / 2}$ of similar magnitude [7, $16,18]$.

In the population pharmacokinetic model, bendamustine declined from $C_{\max }$ in a triphasic manner, with an intermediate elimination $t_{1 / 2}$ of $\sim 40 \mathrm{~min}$ and a slow terminal $t_{1 / 2}$ of $\sim 110 \mathrm{~h}$ [17]. The last phase was only measured after the introduction of advanced technology that allowed for detection of very low concentrations of bendamustine. Because the terminal elimination phase represents a small portion of the overall systemic exposure of bendamustine $(<1 \%)$, the intermediate $t_{1 / 2}$ is considered the effective $t_{1 / 2}$ of bendamustine [17]. The human mass balance study showed a similar rapid initial distribution phase and effective $t_{1 / 2}$. Although the slow terminal phase was not observed in the mass balance study, this was probably due to the fivefold higher lower limit of quantitation for the method used [18].

Concentrations of M3 and M4 declined in biphasic and monophasic manners with elimination $t_{1 / 2}$ of $\sim 3$ and $\sim 0.7 \mathrm{~h}$ (and plasma levels generally undetectable by $\sim 13$ and $\sim 5 \mathrm{~h}$ ), respectively, in the population pharmacokinetic model [17]. In the human mass balance study, the elimination $t_{1 / 2}$ for M3 and M4 were similar ( 1.6 and $\left.\sim 0.5 \mathrm{~h}\right)$ [18].

During the 168 -h period, after the infusion of $\left[{ }^{14} \mathrm{C}\right] \mathrm{ben}$ damustine in the human mass balance study, the mean total recovery of radioactivity in excreta was $76 \%$, of which $\sim 50 \%$ was recovered in urine and $\sim 25 \%$ in feces. In $24 \mathrm{~h}$ after the start of the infusion, $\sim 3 \%$ of the dose was recovered in urine as bendamustine, $<1 \%$ each was recovered as M3 and M4, and 5\% was recovered as HP2 [18, 23]. The high and persistent levels of total radioactivity in urine ( $\sim 36 \%$ of radiochemical dose after $24 \mathrm{~h}$ ) and plasma (mean 
terminal $t_{1 / 2}$ of $197 \mathrm{~h}$ after $168 \mathrm{~h}$ ) compared with those of bendamustine, M3, M4, and HP2 are not uncommon for alkylating agents [25] and indicate the presence of additional longer-lived adducts. As shown in the human mass balance study, bendamustine is extensively metabolized, primarily via non-enzymatic hydrolysis. Renal or hepatic impairment would, therefore, not be expected to have an important effect on the systemic exposure to bendamustine, due to the short $t_{1 / 2}$, dosing schedule, primary metabolic pathways, and the very low renal excretion of bendamustine $[18,23]$.

\section{Comparison of adult and pediatric pharmacokinetic profiles}

At present, bendamustine is not indicated for the treatment of pediatric acute leukemia, but the pharmacokinetic profiles between adult and pediatric populations are similar.

Bendamustine monotherapy was recently investigated in an international, open-label, single-arm, multicenter, phase $1 / 2$ study of heavily pretreated pediatric patients aged 1-20 years with relapsed/refractory acute lymphoblastic leukemia (ALL) or acute myeloid leukemia (AML) [26]. Secondary study objectives included determining the pediatric pharmacokinetic profile of bendamustine relative to the adult pharmacokinetic profile [27].

Systemic exposure to bendamustine $120 \mathrm{mg} / \mathrm{m}^{2}$ in children was similar to that previously reported in the phase 3 adult NHL study $[17,27]$. In the $120 \mathrm{mg} / \mathrm{m}^{2}$ pediatric group $(n=38)$, mean $C_{\max }$ was $6806 \mathrm{ng} / \mathrm{mL}$ (mean $t_{\max }, 1.1 \mathrm{~h}$ ) and mean area under the curve (AUC) $)_{0-24}$ was $8240 \mathrm{ng} \mathrm{h} / \mathrm{mL}$, compared with a mean $C_{\max }$ of $5746 \mathrm{ng} /$ $\mathrm{mL}$ and mean $\mathrm{AUC}_{0-24}$ of $7121 \mathrm{ng} \mathrm{h} / \mathrm{mL}$ in adults $(n=78)$ $[26,27]$.

As with adults, the $C_{\max }$ of bendamustine $120 \mathrm{mg} / \mathrm{m}^{2}$ in pediatric patients was reached by the end of infusion $(\sim 1 \mathrm{~h})$. The pediatric pharmacokinetic profile for bendamustine showed a very rapid distribution phase after the peak plasma concentration, followed by a somewhat slower drug elimination phase. The third phase decline, which has been observed in adults and represents $<1 \%$ of overall AUC, could not be adequately shown in the pediatric study due to limited sampling (no samples were collected in the 12-24 h timeframe, with few samples at later time points) [27].

\section{Bendamustine dosing paradigm}

Bendamustine dosing is based on body surface area (BSA) to reduce interindividual variability in drug

\section{a Adults-AUC Versus Administered Dose}
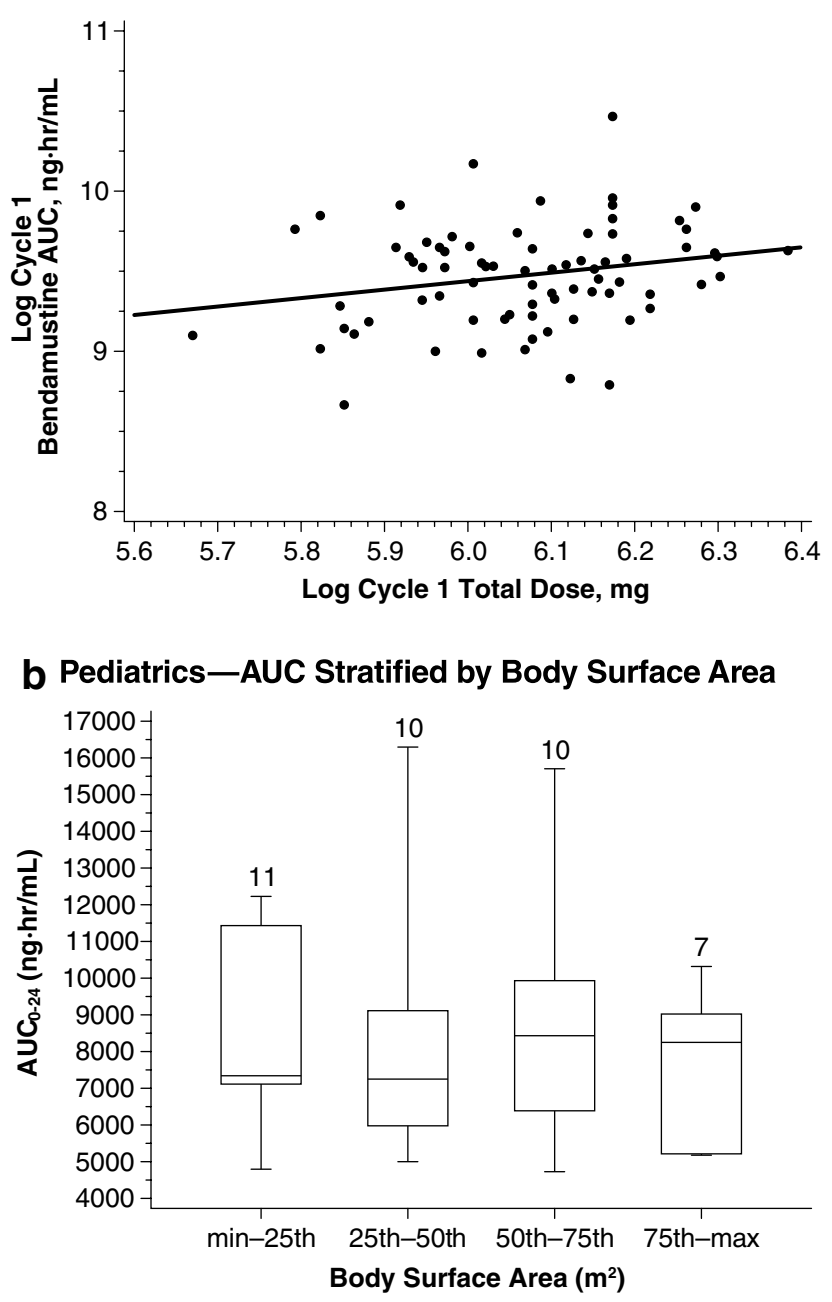

Fig. 2 Effect of body surface area on systemic exposure. a The line represents a linear regression. b Boxes are 25th, 50th, and 75th percentiles; whiskers are 5th and 95th percentiles. The numbers above the box represent the number of patients. Pediatrics panel: adapted with permission of Informa Healthcare [27]

concentrations and to achieve comparable systemic exposure across patients. Recent data confirm the appropriateness of a BSA-based dosing scheme for bendamustine [27].

A population pharmacokinetic analysis in 43 pediatric patients with acute leukemia who received bendamustine $\left(120 \mathrm{mg} / \mathrm{m}^{2}, n=38 ; 90 \mathrm{mg} / \mathrm{m}^{2}, n=5\right)$ in the phase $1 / 2$ pediatric study demonstrated comparable systemic exposure and little difference $(<15 \%)$ in median bendamustine AUC and $C_{\max }$ across BSA quartiles, despite a wide range of BSAs $\left(0.49-1.86 \mathrm{mg} / \mathrm{m}^{2}\right)$ (Fig. 2) [27]. No dose-limiting toxicities were reported [26]. Because systemic exposure to bendamustine $120 \mathrm{mg} / \mathrm{m}^{2}$ was similar between adult and pediatric patients-with mean $\mathrm{AUC}_{0-24}$ and $C_{\max }$ values $<16 \%$ higher in the pediatric patients 
population-higher doses were not assessed in the pediatric study, per protocol $[17,27]$.

\section{Effect of selected covariates on the pharmacokinetics of bendamustine in adult and pediatric patients}

The potential impact of age, sex, race, and hepatic or renal impairment on the pharmacokinetics of bendamustine has been assessed in both adult and pediatric patients using population pharmacokinetic analysis. Available evidence does not suggest significant differences based on age, sex, or race. Mild hepatic and renal impairment did not show significant effects on the pharmacokinetics of bendamustine; however, some differences in systemic exposure cannot be ruled out.

Within the population pharmacokinetic analysis, covariates were assessed using forward selection and backward elimination procedures [17]. A model was created and individual concentration-time profiles and pharmacokinetic parameters were generated using the Bayesian approach. Individual systemic exposures (expressed as $C_{\max }$ and AUC) were then compared between adult and pediatric patients $[17,27]$. Because data from the adult and pediatric population pharmacokinetic analyses were limited for certain covariates, definitive conclusions regarding the potential impact of those covariates could not be drawn.

\section{Effect of age on systemic exposure to bendamustine}

Overall data from adult and pediatric studies provide evidence that age is not an important determinant of systemic exposure to bendamustine [17, 27].

\section{Adult patients}

In the phase 3 study in adults with NHL, the median bendamustine $C_{\max }$ and AUC showed little difference $(<6 \%)$ among three age groups $(16-64,65-74$, and $\geq 75$ years) following bendamustine $120 \mathrm{mg} / \mathrm{m}^{2}$ (Fig. 3). In a model based on that study, the predicted means for $C_{\max }$ and $\mathrm{AUC}_{0-24}$ across the entire age range were $5746 \mathrm{ng} / \mathrm{mL}$ and $7121 \mathrm{ng} \mathrm{h} / \mathrm{mL}$, respectively [17, 27].

\section{Pediatric patients}

Similarly, median bendamustine AUC and $C_{\max }$ varied by $<20 \%$ across all age groups (1-6, 7-11, and 12-21 years) in the pediatric study (Fig. 3) [27]. Furthermore, mean $C_{\max }$ and $\mathrm{AUC}_{0-24}$ across the entire age range in the pediatric study were $6806 \mathrm{ng} / \mathrm{mL}$ and $8240 \mathrm{ng} \mathrm{h} / \mathrm{mL}$,

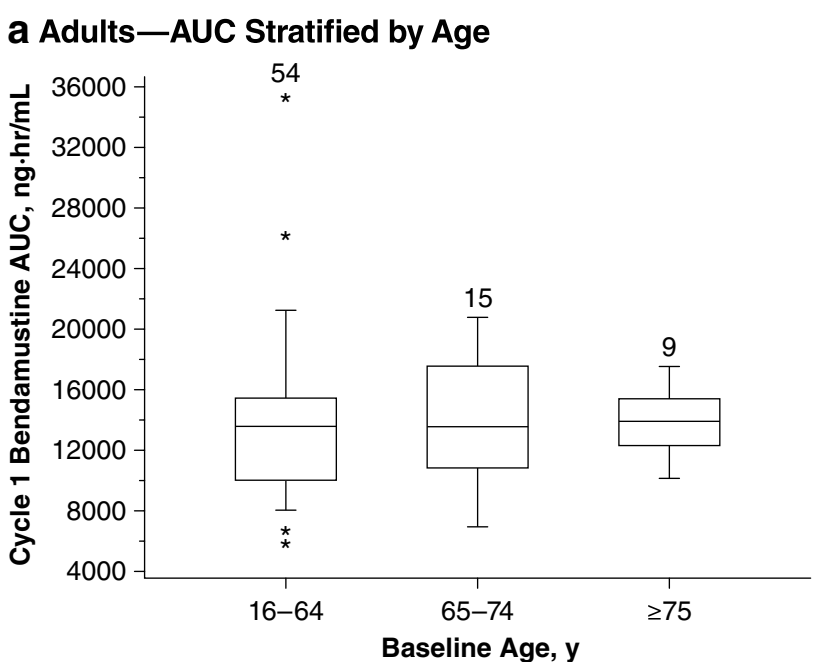

b Pediatrics-AUC Stratified by Age

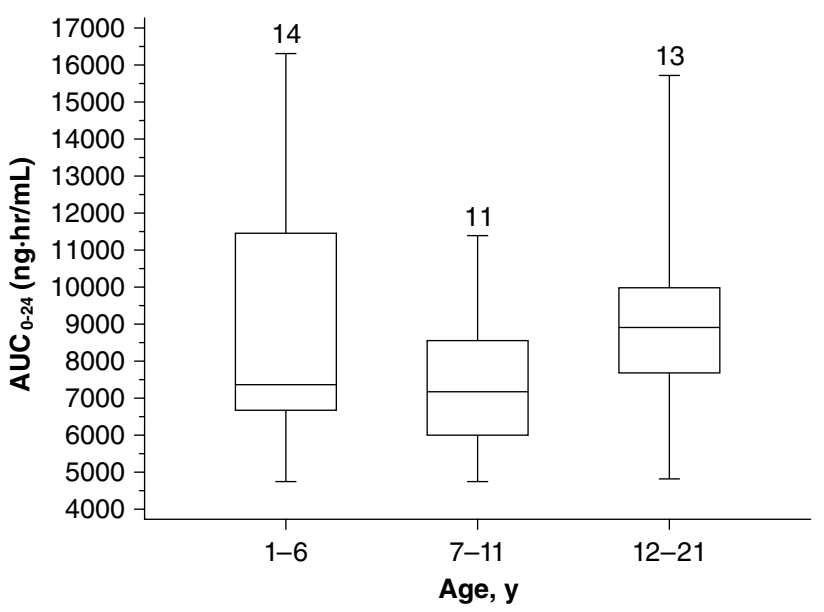

Fig. 3 Effect of age on systemic exposure. Boxes are 25th, 50th, and 75th percentiles; whiskers are 5th and 95th percentiles. Asterisks are data points outside this range. The numbers above the box represent the number of patients. Pediatrics panel: adapted with permission of Informa Healthcare [27]

respectively, which were comparable to those of the adult population [27].

\section{Effect of sex on systemic exposure to bendamustine}

The sex of adult or pediatric patients has been shown to have no significant effect on systemic exposure to bendamustine.

\section{Adult patients}

In the phase 3 adult NHL study, there were no notable differences between median bendamustine AUC and $C_{\max }$ for men and women, which were within $2 \%$ of each other $[17,27]$. 


\section{Pediatric patients}

Sex differences in median bendamustine AUC and $C_{\max }$ levels in the pediatric population pharmacokinetic analysis did not meet the prespecified significance level in the population analysis. However, the levels were lower by 26 and $16 \%$, respectively, in male compared with female patients [27]. The reason for the apparent higher exposure in female patients remains unknown [27].

\section{Effect of race on systemic exposure to bendamustine}

Race appears to have no significant effect on systemic exposure to bendamustine.

\section{Adult patients}

There were too few non-Caucasian patients in the phase 3 NHL study to draw any conclusions regarding the influence of race on bendamustine systemic exposure in adults [17]. However, there was no evidence of any differences in pharmacokinetic properties among the different race groups, including black $(n=5)$, Asian $(n=1)$, and Hispanic $(n=1)$ patients. The pharmacokinetic profiles of 12 Japanese patients with relapsed/refractory NHL or mantle cell lymphoma who received bendamustine $120 \mathrm{mg} / \mathrm{m}^{2}$ alone $\left(n=6 ; C_{\max } 8.6 \mu \mathrm{g} / \mathrm{mL}, \mathrm{AUC}_{0-\mathrm{t}} 10.2 \mu \mathrm{g} \mathrm{h} / \mathrm{mL}\right)$ or in combination with rituximab $\left(n=6 ; C_{\max } 5.4 \mu \mathrm{g} / \mathrm{mL}\right.$, $\mathrm{AUC}_{0-\mathrm{t}} 6.1 \mu \mathrm{g} \mathrm{h} / \mathrm{mL}$ ) were demonstrated to be similar to that of patients in the phase 3 adult NHL study $(n=78$; $C_{\max } 5.8 \mu \mathrm{g} / \mathrm{mL}, \mathrm{AUC}_{0-\mathrm{t}} 13.6 \mu \mathrm{g} \mathrm{h} / \mathrm{mL}$ ) [17, 28, 29].

\section{Pediatric patients}

In the pediatric study of bendamustine $120 \mathrm{mg} / \mathrm{m}^{2}$, median AUC and $C_{\max }$ values in Caucasians $(n=20)$ and Asians ( $n=11$ ) were within $<5 \%$ of each other [27]. Although bendamustine systemic exposure was $\leq 30 \%$ lower in nonCaucasian/non-Asian patients ( $n=7$, most of whom were black or Hispanic) than in Caucasian and Asian patients, the difference did not meet the prespecified level of significance for bendamustine pharmacokinetic parameters.

\section{Effect of hepatic impairment on systemic exposure to bendamustine}

The effect of hepatic impairment on the pharmacokinetics of bendamustine remains to be fully elucidated. Although no significant change in bendamustine clearance has been noted in patients with mild hepatic impairment [7, 17], some differences in bendamustine systemic exposure in this population cannot be ruled out. Due to limited data, the current recommendation is for bendamustine to be used
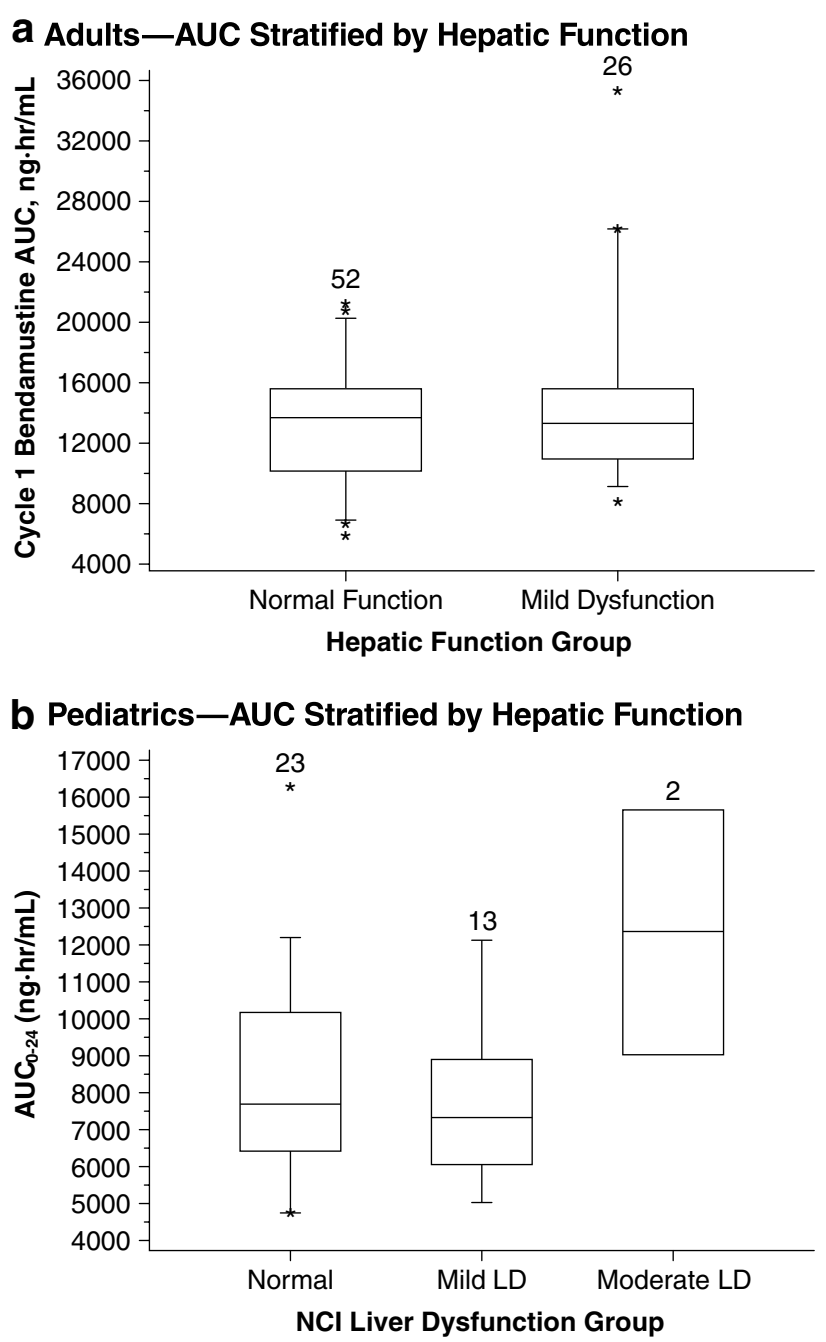

Fig. 4 Effect of hepatic impairment on systemic exposure. Boxes are 25th, 50th, and 75th percentiles; whiskers are 5th and 95th percentiles. Asterisks are data points outside this range. The numbers above the box represent the number of patients. Pediatrics panel: adapted with permission of Informa Healthcare [27]

with caution in patients with mild hepatic impairment and not to be used in patients with moderate hepatic impairment (aspartate aminotransferase [AST] or alanine aminotransferase [ALT] 2.5-10 $\times$ upper limit of normal [ULN] and total bilirubin 1.5-3 $\times$ ULN) or severe hepatic impairment (total bilirubin $>3 \times \mathrm{ULN}$ ) [7].

\section{Adult patients}

In the adult NHL phase 3 study, the pharmacokinetic profile of bendamustine in 26 patients with mild hepatic impairment (defined as total bilirubin $\leq$ ULN, AST $\geq$ ULN to $2.5 \times \mathrm{ULN}$, and/or alkaline phosphatase $\geq \mathrm{ULN}$ to $5.0 \times \mathrm{ULN}$ ) was not substantially different from that in 52 patients with normal function (Fig. 4) [7, 17]. In addition, 
bendamustine tolerability was found to be adequate in a pilot study of six patients with advanced bile duct cancer and substantial hepatic dysfunction (bilirubin $\leq 3 \times \mathrm{UNL}$ ) [30]. Furthermore, two patients with severe liver impairment and aggressive NHL were recently reported to have been successfully treated with bendamustine and rituximab [31]. Their total serum bilirubin levels were $10 \times \mathrm{ULN}$, but improved greatly after treatment; mild-to-moderate increases in ALT and AST levels in both cases also improved following treatment. The liver impairment in both patients was considered obstructive rather than functional [31].

\section{Pediatric patients}

In the pediatric population pharmacokinetic analysis, a difference of $\leq 5 \%$ was observed in the median bendamustine $\mathrm{AUC}_{0-24}$ and $C_{\max }$ in the 23 patients with normal hepatic function and the 13 patients with mild hepatic impairment. Patients with moderate hepatic impairment $(n=2)$ had higher bendamustine systemic exposure (Fig. 4) [27].

\section{Effect of renal impairment on systemic exposure to bendamustine}

The effect of renal impairment on the pharmacokinetics of bendamustine remains to be fully elucidated. Although no significant change in bendamustine clearance has been noted in patients with mild-to-moderate renal impairment [7, 17], some differences in bendamustine systemic exposure in this population cannot be ruled out. Given that only $\sim 3 \%$ of the bendamustine dose is eliminated renally, renal impairment would be unlikely to have a substantive effect on bendamustine systemic exposure [18, 32]. However, due to limited data, the current recommendation is for bendamustine to be used with caution in patients with mild-tomoderate renal impairment and not to be used in patients with creatinine clearance $(\mathrm{CrCL})<40 \mathrm{~mL} / \mathrm{min}$ [7].

\section{Adult patients}

In the adult phase 3 NHL study, there was no meaningful difference in the pharmacokinetics of bendamustine among the 31 patients with mild or moderate renal impairment (CrCL, 30-80 $\mathrm{mL} / \mathrm{min})$ [7, 17] and the 47 patients with normal renal function (Fig. 5). In addition, a myeloma study showed no differences in the plasma kinetics of bendamustine or its metabolites between patients with normal renal function $(n=12)$ and those with renal insufficiency ( $n=12$, including 5 who were under continuous hemodialysis), with only a moderate increase in the frequency of myelotoxicity observed in the renally impaired group, and no dose reductions were required [32].
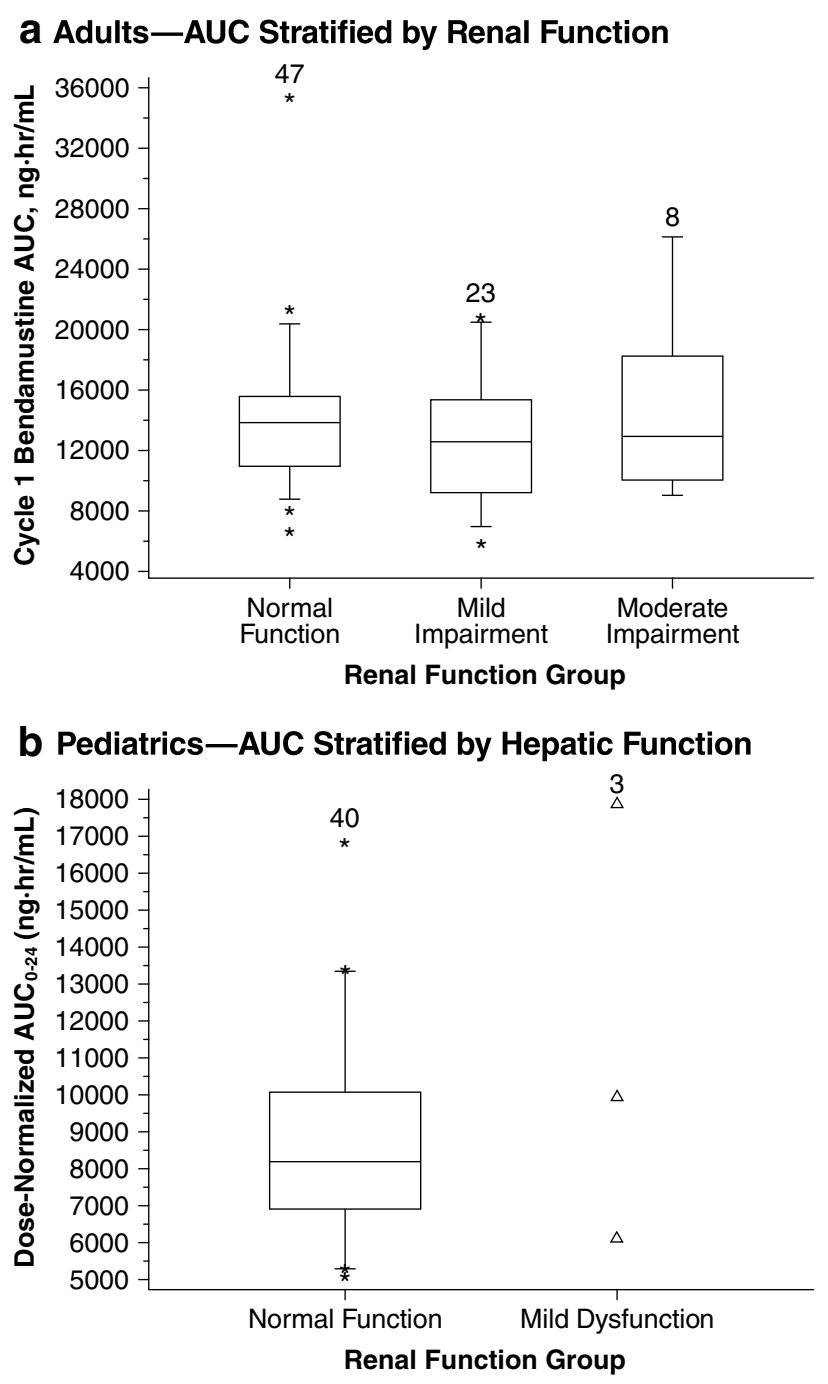

Fig. 5 Effect of renal impairment on systemic exposure. Boxes are 25th, 50th, and 75th percentiles; whiskers are 5th and 95th percentiles. Asterisks are data points outside this range. Triangles show individual data points for patients with mild renal dysfunction. The numbers above the box represent the number of patients. Pediatrics panel: adapted with permission of Informa Healthcare [27]

A retrospective safety assessment in NHL and CLL of 104 renally impaired patients $(\mathrm{CrCL}$ of $<40 \mathrm{~mL} / \mathrm{min})$ and 836 patients with $\mathrm{CrCL} \geq 40 \mathrm{~mL} / \mathrm{min}$ showed no significant differences in laboratory toxicities between the CrCL groups [33]. Renally impaired patients were found to have a twofold increase in the risk of two evaluated grade 3-4 adverse events compared with patients who had a CrCL $\geq 40 \mathrm{~mL} / \mathrm{min}$ : increased blood urea nitrogen for CLL and NHL together $(P=0.02)$, and thrombocytopenia in a subanalysis of NHL patients with a $\mathrm{CrCL}<40 \mathrm{~mL} / \mathrm{min}$ versus those with NHL and a CrCL $\geq 60 \mathrm{~mL} / \mathrm{min}(P=0.025)$ [33]. Likewise, in two prospective clinical studies [34, 35] and one retrospective study [36] of myeloma patients with 
moderate-to-severe renal impairment or renal failure/dialysis, bendamustine in combination with other drugs (prednisone and bortezomib, or thalidomide and dexamethasone) was well tolerated.

\section{Pediatric patients}

In pediatric patients, no differences in dose-normalized bendamustine pharmacokinetics were observed between those with mild renal impairment $(n=3)$, as defined by glomerular filtration rate per National Kidney Foundation 2002 age-based guidelines [37], and normal renal function $(n=40)$ (Fig. 5) [27].

\section{Potential for CYP interactions with bendamustine}

Because bendamustine is primarily biotransformed via hydrolysis [7, 14, 23], there is limited potential for direct drug interaction.

Based on in vitro data, bendamustine has a low potential to affect drug metabolism via human cytochrome $\mathrm{P} 450$ enzymes. At concentrations up to $200 \mu \mathrm{M}$, bendamustine did not inhibit the metabolism of substrates specific for isoenzymes CYP1A2, CYP2C9/10, CYP2D6, CYP2E1, or CYP3A4/5, and at up to $100 \mu \mathrm{M}$, it showed no potential for induction of CYP enzymes [7].

However, the M3 and M4 metabolites, both of which make little contribution to the cytotoxicity of bendamustine $[7,16,18]$, are formed by CYP1A2 [16]. As a result, systemic exposure to bendamustine in the presence and absence of CYP1A2 inhibitors and inducers was evaluated. A comparison between the observed bendamustine concentration-time profile following coadministration with a CYP1A2 inhibitor (e.g., allopurinol, famotidine, ranitidine, or ciprofloxacin) in 15 patients or with a CYP1A2 inducer in two patients was similar to that following administration without a CYP1A2 inhibitor/inducer, which confirms that oxidative metabolism by CYP1A2 is a relatively minor contributor to the elimination of bendamustine (Fig. 6).

\section{Bendamustine exposure-response relationship}

Data suggest that $C_{\max }$ is an essential component of the activity of bendamustine.

The excess of B cells associated with CLL is caused by a decrease in apoptosis rather than an increase in cell proliferation [38-41]. In vitro, bendamustine has been shown to induce apoptosis in a dose- and time-dependent manner in B-CLL lymphocytes, and elevated plasma concentrations seem to be more relevant than prolonged exposure [42]. Ex vivo studies conducted to assess the effect of

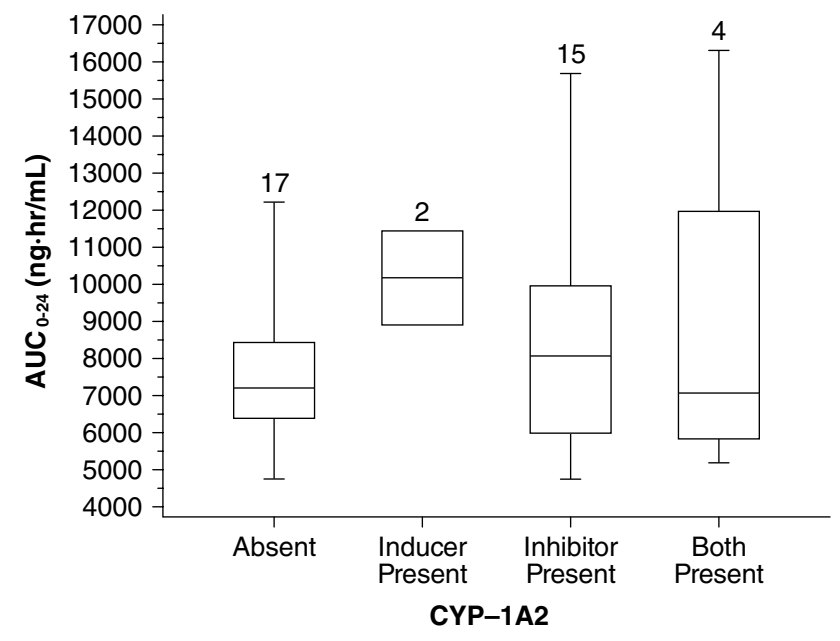

Fig. 6 Pharmacokinetics in presence or absence of CYP1A2 inhibitors/ inducers. Boxes are 25th, 50th, and 75th percentiles; whiskers are 5th and 95th percentiles. The numbers above the box represent the number of patients. Adapted with permission of Informa Healthcare [27]

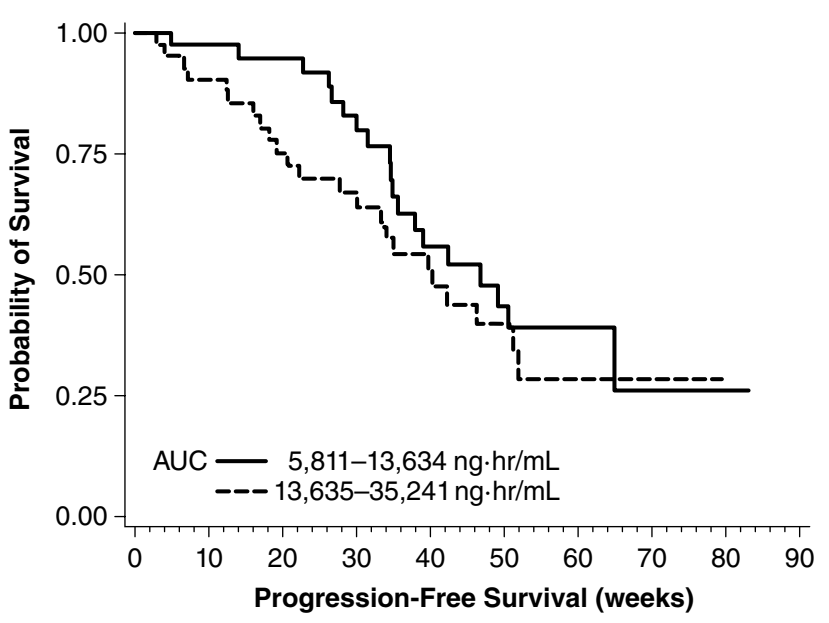

Fig. 7 Kaplan-Meier plot of PFS, stratified by median bendamustine AUC. With kind permission from Springer Science+Business Media: Figure 5 [17]

bendamustine on leukemic cells in CLL have shown that the median lethal dose $\left(\mathrm{LD}_{50}\right)$ of bendamustine is $4.3 \mu \mathrm{g} /$ $\mathrm{mL}$ in cells from previously treated patients and $7.4 \mu \mathrm{g} /$ $\mathrm{mL}$ in cells from previously untreated patients [42]. In the adult relapsed/refractory NHL phase 3 study, bendamustine $120 \mathrm{mg} / \mathrm{m}^{2}$ resulted in a peak exposure of $\sim 6 \mu \mathrm{g} / \mathrm{mL}$ (within the $\mathrm{LD}_{50}$ range) [17].

\section{Adult patients with NHL}

The pharmacokinetic profile of bendamustine and exposure-response relationships were described in 80 patients in the adult NHL phase 3 trial who received bendamustine 
$120 \mathrm{mg} / \mathrm{m}^{2}$ [17]. Eighty-five percent of the patients had at least a partial response following treatment with bendamustine, but there were no significant associations between any of the measures of exposure (e.g., bendamustine AUC and $C_{\max }$ ) and treatment response or duration of response. A separate trend was noted in progression-free survival based on bendamustine AUC value above and below the median value $(P=0.3025$; Fig. 7$)$.

\section{Pediatric patients with acute leukemia}

The population pharmacokinetic and pharmacodynamic analysis in 43 heavily pretreated pediatric patients explored the exposure-response relationship in the 38 patients who received single-agent bendamustine at $120 \mathrm{mg} / \mathrm{m}^{2}$ and were evaluable for efficacy in this dose-ranging/safety study [27]. Of the 22 patients receiving $120 \mathrm{mg} / \mathrm{m}^{2}$ with a defined best overall response, $9 \%$ achieved a partial response, $32 \%$ had stable disease, and $59 \%$ had progressive disease. The remaining five patients received bendamustine $90 \mathrm{mg} /$ $\mathrm{m}^{2}$, two of whom achieved a complete response (both with ALL) [26]. Although no clear exposure-response relationship was observed in the efficacy analysis [27], two ALL patients with partial response as their best overall response had slightly higher bendamustine AUC and $C_{\max }$ values than the median systemic exposures of patients with stable or progressive disease [26]. No patients with AML had a response. Furthermore, the median bendamustine AUC and $C_{\max }$ for the $16 \mathrm{AML}$ patients were 17 and $16 \%$ lower, respectively, than for the 22 ALL patients. Response data for the study population suggest that single-agent bendamustine has some activity in heavily pretreated patients with relapsed and refractory ALL, but not in AML [27].

\section{Bendamustine safety-exposure relationships}

The relationship between bendamustine systemic exposure and specific adverse events during treatment was evaluated in the adult and pediatric population pharmacokinetic analyses [17, 27]. Significant correlations were observed only for nausea in the adult population and infection (e.g., aspergillosis, paronychia, sinusitis, and staphylococcal infection) in the pediatric population. It should be noted that although nausea was evaluated in both studies, it was not shown to be associated with bendamustine systemic exposure in pediatric patients.

\section{Adult patients with NHL}

The correlation between exposure and safety was reported in 80 patients who received bendamustine $120 \mathrm{mg} / \mathrm{m}^{2}$ in the adult NHL phase 3 trial [17]. Among the five safety endpoints of interest (i.e., neutropenia, thrombocytopenia, nausea, vomiting, and fatigue), only nausea was found to be significantly correlated with bendamustine $C_{\max }$ $(P=0.013)$, with the probability of nausea increasing along with bendamustine $C_{\max }$.

The high rate of prophylactic antiemetic use $(80 \%)$ prevented the statistical analysis of the influence of their use on the association between bendamustine exposure and nausea [17].

\section{Pediatric patients with acute leukemia}

In the population pharmacokinetic and pharmacodynamic safety analysis in 43 pediatric patients, infection was the only adverse event that was shown to be significantly correlated with bendamustine $C_{\max }(P<0.5)$. The most common infections were aspergillosis $\left(n=2,120 \mathrm{mg} / \mathrm{m}^{2}\right)$, sinusitis $\left(n=1\right.$ each, 90 and $\left.120 \mathrm{mg} / \mathrm{m}^{2}\right)$, and staphylococcal infection $\left(n=2,120 \mathrm{mg} / \mathrm{m}^{2}\right)$. The most common grade $3 / 4$ infections were the two patients with aspergillosis and two with staphylococcal infection. As expected, the risk of developing an infection was greater in the absence of prophylactic antibiotic use $(n=9)$. No other exposure measures were a significant predictor of developing an infection [27].

\section{Potential for drug-drug interactions between bendamustine and monoclonal antibodies}

Although not currently approved as combination therapy, bendamustine has demonstrated improved overall response rates and progression-free survival when combined with rituximab and/or other chemotherapeutic agents in the treatment of lymphoid malignancies [9, 43-45]. Based on the pharmacokinetic characteristics of bendamustine and rituximab, a drug-drug interaction would not typically be expected. However, no formal clinical pharmacology study has been conducted to specifically assess pharmacokinetic interactions between bendamustine and other drugs. Furthermore, there are limited data on the pharmacokinetics of rituximab when combined with other drugs and on variables influencing individual exposure [46-48].

As noted before, bendamustine is a small molecule that is approximately $95 \%$ bound to plasma proteins, mainly albumin [7, 19], and is primarily metabolized via hydrolysis $[7,14,23]$. In contrast, rituximab is a large molecule with targeted binding to CD20 antigen (but not to plasma proteins, such as albumin); a low volume of distribution (<3 L); and distinct elimination pathways that include hepatic proteolysis, the reticuloendothelial system, targetmediated elimination, and endocytosis [49-51]. However, the possibility of drug-drug interactions between 
bendamustine and rituximab cannot be completely ruled out. Indeed, indirect pharmacokinetic interactions between small molecules and monoclonal antibodies have been reported [48].

Two recent studies and data from the literature indicate that the potential for a drug-drug interaction between bendamustine and rituximab is low [52]. One of the studies was an open-label, multicenter, phase 3 study in adults who received bendamustine-rituximab combination therapy for advanced indolent NHL or mantle cell lymphoma [52]. The other study, which served as the data source for the bendamustine population pharmacokinetic model used in the combination therapy study, was the aforementioned phase 3 NHL study in adults who received bendamustine monotherapy [17].

\section{Bendamustine-rituximab combination therapy study}

In the bendamustine-rituximab combination therapy study, patients received rituximab $\left(375 \mathrm{mg} / \mathrm{m}^{2}\right)$ followed by bendamustine $\left(90 \mathrm{mg} / \mathrm{m}^{2}\right)$ on day 1 of each cycle and bendamustine $\left(90 \mathrm{mg} / \mathrm{m}^{2}\right)$ on day 2 of each cycle. The final analysis dataset included bendamustine concentration samples from 49 patients and rituximab concentration samples from 19 patients [52].

\section{Bendamustine monotherapy study}

In the bendamustine monotherapy study, patients received bendamustine $\left(120 \mathrm{mg} / \mathrm{m}^{2}\right)$ on days 1 and 2 of each cycle. Bendamustine plasma concentrations from 78 adult patients were described in a three-compartment, open population pharmacokinetic model with zero-order input and first-order elimination [17, 52].

\section{Effect of rituximab on bendamustine pharmacokinetics}

Model-predicted Bayesian estimates of bendamustine clearance showed similar clearance values in patients who received bendamustine with or without rituximab, with median differences within $3.4 \%$ of each other (32.9 vs. $31.8 \mathrm{~L} / \mathrm{h}$, respectively). The two groups did not differ significantly in their log-transformed clearance values (twosided Wilcoxon signed rank test, $P>0.93$ ) [52]. This finding is consistent with results of two small Japanese studies, in which the pharmacokinetic profile of bendamustine monotherapy was observed to be comparable with that of bendamustine in the presence of rituximab $[28,29]$.

\section{Effect of bendamustine on rituximab pharmacokinetics}

Comparison between observed serum rituximab concentrations from the bendamustine-rituximab combination therapy study and those in four publications on the pharmacokinetics of rituximab (without bendamustine) in six different populations suggests that bendamustine does not affect the systemic exposure to rituximab [52-56]. For all studies, rituximab concentrations were compared at end of infusion (in the bendamustine-rituximab study, this was prior to bendamustine infusion, i.e., rituximab alone), $24 \mathrm{~h}$, and 7 days post infusion. Median observed serum rituximab concentrations, in the absence (end of infusion) and presence of bendamustine ( $24 \mathrm{~h}$ and 7 days post infusion), in the bendamustine-rituximab combination therapy study were consistently lower than median weighted averages from literature data by 24,30 , and $53 \%$ at each respective time point. However, the relative changes in serum concentrations over time were generally consistent across the studies [52-56]. Disparities in technique (e.g., duration of rituximab intravenous infusion, assay methodology, or assay sensitivity) could have resulted in the differences between the rituximab monotherapy findings across the studies [52].

\section{Conclusions}

Maximal concentrations of bendamustine are typically reached at the end of infusion $(\sim 1 \mathrm{~h})$, with rapid elimination characterized by an effective $t_{1 / 2}$ of $\sim 40 \mathrm{~min}$ and with no expected accumulation after multiple daily doses [7, $14,17]$. The compound is rapidly distributed to the site of action, but not extensively distributed into tissues [7, 17]. It primarily undergoes hydrolytic metabolism (without hepatic enzymes), into HP1 and HP2 metabolites, which have little or no activity [7, 14]. The active metabolites, M3 and M4, are formed via a hepatic CYP1A2 oxidative pathway [16]; however, their contributions to the cytotoxic effect of bendamustine is minimal $[7,16,18]$. In addition, renal elimination is minor; only $\sim 3 \%$ of a bendamustine dose is excreted in urine [18, 32].

Systemic exposure to bendamustine is similar in adults and pediatric patients $[17,27]$, which confirms the appropriateness of BSA-based dosing [27]. Age, sex, and race have minimal effects on the systemic exposure to bendamustine [7, 17, 27].

Bendamustine is not easily displaced by and does not displace other highly protein-bound drugs [19] and has a low likelihood of direct or indirect drug-drug interactions [52]. Based on in vitro and clinical data, $C_{\max }$ seems to be an important predictor of response to bendamustine [17, 42]. No clear dose-response relationship to efficacy has been observed, while higher doses may be associated with increased risk of nausea or infection [17, 27].

Bendamustine is a unique alkylator with demonstrated efficacy in NHL and CLL as well as clinical activity in a 
wide range of other malignancies [1-7]. Clinical experience with bendamustine has been extensive, and, together with its overall pharmacokinetics, pharmacokinetic/pharmacodynamic relationships, and drug-drug interactions, support the appropriateness of a BSA-based dosing scheme for a wide range of patient populations.

Acknowledgments This research was sponsored by and conducted by Teva Branded Pharmaceutical Products R\&D Inc., Frazer, PA. Funding for editorial, design, and production support was provided by Teva Branded Pharmaceutical Products R\&D, Inc., to The Curry Rockefeller Group, LLC, Tarrytown, NY.

Conflict of interest At the time this study was conducted, M. Darwish was a salaried employee at Teva Branded Pharmaceutical Products R\&D, Inc. M. Bond, E. Hellriegel, P. Robertson, Jr., and J. P. Chovan are employed by Teva Branded Pharmaceutical Products R\&D, Inc.; Hellriegel and Robertson, Jr., own stock/options in the company.

Open Access This article is distributed under the terms of the Creative Commons Attribution License which permits any use, distribution, and reproduction in any medium, provided the original author(s) and the source are credited.

\section{References}

1. Leoni LM, Bailey B, Reifert J et al (2008) Bendamustine (Treanda) displays a distinct pattern of cytotoxicity and unique mechanistic features compared with other alkylating agents. Clin Cancer Res 14:309-317

2. Fowler N, Kahl BS, Lee P et al (2011) Bortezomib, bendamustine, and rituximab in patients with relapsed or refractory follicular lymphoma: the phase II VERTICAL study. J Clin Oncol 29:3389-3395

3. Friedberg JW, Cohen P, Chen L et al (2008) Bendamustine in patients with rituximab-refractory indolent and transformed non-Hodgkin's lymphoma: results from a phase II multicenter, single-agent study. J Clin Oncol 26:204-210

4. Pönisch W, Rozanski M, Goldschmidt $\mathrm{H}$ et al (2008) Combined bendamustine, prednisolone and thalidomide for refractory or relapsed multiple myeloma after autologous stem-cell transplantation or conventional chemotherapy: results of a phase I clinical trial. Br J Haematol 143:191-200

5. Ohmachi K, Ando K, Ogura M et al (2010) Multicenter phase II study of bendamustine for relapsed or refractory indolent B-cell non-Hodgkin lymphoma and mantle cell lymphoma. Cancer Sci 101:2059-2064

6. Warsch S, Hosein PJ, Maeda LS, Alizadeh AA, Lossos IS (2012) A retrospective study evaluating the efficacy and safety of bendamustine in the treatment of mantle cell lymphoma. Leuk Lymphoma 53:1299-1305

7. Teva Branded Pharmaceutical Products R\&D, Inc (2013) TREANDA $^{\circledR}$ (bendamustine hydrochloride) prescribing information http://treandahcp.com/PDF/TREANDA_final_PI.pdf. Accessed 28 Oct 2014

8. Knauf WU, Lissichkov T, Aldaoud A et al (2009) Phase III randomized study of bendamustine compared with chlorambucil in previously untreated patients with chronic lymphocytic leukemia. J Clin Oncol 27:4378-4384

9. Robinson KS, Williams ME, van der Jagt RH et al (2008) Phase II multicenter study of bendamustine plus rituximab in patients with relapsed indolent B-cell and mantle cell non-Hodgkin's lymphoma. J Clin Oncol 26:4473-4479

10. Strumberg D, Harstrick A, Doll K, Hoffmann B, Seeber S (1996) Bendamustine hydrochloride activity against doxorubicinresistant human breast carcinoma cell lines. Anticancer Drugs 7:415-421

11. Ozegowski W, Krebs D (1971) IMET 3393, $\gamma$-[1-methyl-5bis( $\beta$-chloroethyl)-amino-benzimidazolyl-2]-butyric acid hydrochloride, a new cytostatic agent from among the series of benzimidazole mustard compounds. Zbl Pharm 110:1013-1019 [in German]

12. Hirschberg E, Gellhorn A, Gump WS (1957) Laboratory evaluation of a new nitrogen mustard, 2-[di-(2-chloroethyl)aminomethyl]benzimidazole, and of other 2-chloroethyl compounds. Cancer Res 17:904-910

13. Woolley DW (1944) Some biological effects produced by benzimidazole and their reversal by purines. J Biol Chem 152:225-232

14. Preiss R, Sohr R, Matthias M, Brockmann B, Huller H (1985) The pharmacokinetics of bendamustine (Cytostasane) in humans. Pharmazie 40:782-784

15. Weber H, Amlacher R, Preiss R, Hoffmann H (1991) Pharmacokinetics of bendamustine (Cytostasan) in B6D2F1-mice. Pharmazie 46:589-591 [in German]

16. Teichert J, Baumann F, Chao Q et al (2007) Characterization of two phase I metabolites of bendamustine in human liver microsomes and in cancer patients treated with bendamustine hydrochloride. Cancer Chemother Pharmacol 59:759-770

17. Owen JS, Melhem M, Passarell JA, D'Andrea D, Darwish M, Kahl B (2010) Bendamustine pharmacokinetic profile and exposure-response relationships in patients with indolent non-Hodgkin's lymphoma. Cancer Chemother Pharmacol 66:1039-1049

18. Dubbelman A-C, Rosing H, Darwish M et al (2013) Pharmacokinetics and excretion of ${ }^{14} \mathrm{C}$-bendamustine in patients with relapsed or refractory malignancy. Drugs R D 13:17-28

19. Haase D, Preiss R, Sohr R (1990) Untersuchungen zur plasmaeiweisbindung von bendamustin (Cytostasan) und ambazone. $\mathrm{Z}$ Klin Med 45:1267-1271

20. Bezek S, Kukan M, Scasnár V, Lukácsová M, Trnovec T (1996) Selective uptake of the anticancer drug bendamustine by liver and kidney tissues following its intravenous administration to mice. Methods Find Exp Clin Pharmacol 18:117-122

21. Kanekal S, Crain B, Elliott G (2004) SDX-105 (Treanda ${ }^{\mathrm{TM}}$ ) enhances the tumor growth inhibitory effect of rituximab in Daudi lymphoma xenografts. Blood (ASH Annual Meeting Abstracts) 104:229b. [Abstract 4580]

22. Ackler S, Mitten MJ, Chen J et al (2012) Navitoclax (ABT263) and bendamustine \pm rituximab induce enhanced killing of non-Hodgkin's lymphoma tumours in vivo. Br J Pharmacol 167:881-891

23. Dubbelman A-C, Jansen RS, Rosing H, Darwish M, Hellriegel E, Robertson P Jr, Schellens JHM, Beijnen JH (2012) Metabolite profiling of bendamustine in urine of cancer patients after administration of $\left[{ }^{14} \mathrm{C}\right]$ bendamustine. Drug Metab Dispos 40:1297-1307

24. Chovan JP, Li F, Yu E, Ring SC (2007) Metabolic profile of $\left[{ }^{14} \mathrm{C}\right]$ bendamustine in rat urine and bile: preliminary structural identification of metabolites. Drug Metab Dispos 35:1744-1753

25. Beumer JH, Beijnen JH, Schellens JH (2006) Mass balance studies, with a focus on anticancer drugs. Clin Pharmacokinet 45:33-58

26. Fraser C, Brown P, Megason G et al (2014) Open-label bendamustine monotherapy for pediatric patients with relapsed or refractory acute leukemia: efficacy and tolerability. J Pediatr Hematol Oncol 36:e212-e218

27. Darwish M, Megason G, Bond M, Hellriegel E, Robertson P Jr, Grasela T, Phillips L (2014) Population pharmacokinetics and 
pharmacokinetic/pharmacodynamics of bendamustine in pediatric patients with relapsed/refractory acute leukemia. Curr Med Res Opin 30(11):2305-2315. doi:10.1185/03007995.2014.9419 76

28. Ogura M, Uchida T, Taniwaki M et al (2010) Phase I and pharmacokinetic study of bendamustine hydrochloride in relapsed or refractory indolent B-cell non-Hodgkin lymphoma and mantle cell lymphoma. Cancer Sci 101:2054-2058

29. Ogura M, Ando K, Taniwaki M et al (2011) Feasibility and pharmacokinetic study of bendamustine hydrochloride in combination with rituximab in relapsed or refractory aggressive B cell non-Hodgkin's lymphoma. Cancer Sci 102:1687-1692

30. Schoppmeyer K, Kreth F, Wiedmann M, Mössner J, Preiss R, Caca K (2007) A pilot study of bendamustine in advanced bile duct cancer. Anticancer Drugs 18:697-702

31. McCloskey JK, Broome CM, Cheson BD (2013) Safe and effective treatment of aggressive non-Hodgkin lymphoma with rituximab and bendamustine in patients with severe liver impairment. Clin Adv Hematol Oncol 11:184-188

32. Preiss R, Teichert J, Pönisch W, Niederwieser D, Matthias M, Merkle K (2003) Bendamustine in patients with multiple myeloma and renal insufficiency. Onkologie 26:S131 [Abstract P717]

33. Nordstrom BL, Knopf KB, Teltsch DY, Engle R, Beygi H, Sterchele JA (2014) The safety of bendamustine in patients with chronic lymphocytic leukemia or non-Hodgkin lymphoma and concomitant renal impairment: a retrospective electronic medical record database analysis. Leuk Lymphoma 55:1266-1273. doi: 10.3109/10428194.2013.836600

34. Pönisch W, Andrea M, Wagner I et al (2012) Successful treatment of patients with newly diagnosed/untreated multiple myeloma and advanced renal failure using bortezomib in combination with bendamustine and prednisone. J Cancer Res Clin Oncol 138:1405-1412

35. Pönisch W, Moll B, Bourgeois M et al (2013) Bendamustine and prednisone in combination with bortezomib (BPV) in the treatment of patients with relapsed or refractory multiple myeloma and light chain-induced renal failure. J Cancer Res Clin Oncol 139:1937-1946

36. Ramasamy K, Hazel B, Mahmood S, Corderoy S, Schey S (2011) Bendamustine in combination with thalidomide and dexamethasone is an effective therapy for myeloma patients with end stage renal disease. Br J Haematol 155:632-634

37. National Kidney Foundation, Inc (2002) Part 4. Definition and classification of stages of chronic kidney disease. Am J Kidney Dis 39:S46-S75

38. Gale RP, Caligaris-Cappio F, Dighiero G, Keating M, Montserrat E, Rai K (1994) Recent progress in chronic lymphocytic leukemia. International workshop on chronic lymphocytic leukemia. Leukemia 8:1610-1614

39. Wolowiec D, Benchaib M, Pernas P et al (1995) Expression of cell cycle regulatory proteins in chronic lymphocytic leukemias. Comparison with non-Hodgkin's lymphomas and non-neoplastic lymphoid tissue. Leukemia 9:1382-1388

40. Delmer A, Ajchenbaum-Cymbalista F, Tang R et al (1995) Overexpression of cyclin D2 in chronic B-cell malignancies. Blood 85:2870-2876

41. Vrhovac R, Delmer A, Tang R, Marie J-P, Zittoun R, Ajchenbaum-Cymbalista F (1998) Prognostic significance of the cell cycle inhibitor p27Kip1 in chronic B-cell lymphocytic leukemia. Blood 91:4694-4700
42. Schwänen C, Hecker T, Hübinger G et al (2002) In vitro evaluation of bendamustine induced apoptosis in B-chronic lymphocytic leukemia. Leukemia 16:2096-2105

43. Rummel MJ, Al-Batran SE, Kim SZ et al (2005) Bendamustine plus rituximab is effective and has a favorable toxicity profile in the treatment of mantle cell and low-grade non-Hodgkin's lymphoma. J Clin Oncol 23:3383-3389

44. Rummel MJ, Niederle N, Maschmeyer G et al (2013) Bendamustine plus rituximab versus $\mathrm{CHOP}$ plus rituximab as first-line treatment for patients with indolent and mantle-cell lymphomas: an open-label, multicentre, randomised, phase 3 non-inferiority trial. Lancet 381:1203-1210

45. Flinn IW, van der Jagt R, Kahl BS et al (2014) Open-label, randomized, noninferiority study of bendamustine-rituximab or $\mathrm{R}-\mathrm{CHOP} / \mathrm{R}-\mathrm{CVP}$ in first-line treatment of advanced indolent NHL or MCL: the BRIGHT study. Blood 123:2944-2952

46. Blasco H, Chatelut E, de Bretagne IB, Congy-Jolivet N, Le Guellec C (2009) Pharmacokinetics of rituximab associated with CHOP chemotherapy in B-cell non-Hodgkin's lymphoma. Fundam Clin Pharmacol 23:601-608

47. Cartron G, Blasco H, Paintaud G, Watier H, Le Guellec C (2007) Pharmacokinetics of rituximab and its clinical use: thought for the best use? Crit Rev Oncol Hematol 62:43-52

48. Seitz K, Zhou H (2007) Pharmacokinetic drug-drug interaction potentials for therapeutic monoclonal antibodies: reality check. J Clin Pharmacol 47:1104-1118

49. Keizer RJ, Huitema AD, Schellens JH, Beijnen JH (2010) Clinical pharmacokinetics of therapeutic monoclonal antibodies. Clin Pharmacokinet 49:493-507

50. Ng CM, Bruno R, Combs D, Davies B (2005) Population pharmacokinetics of rituximab (anti-CD20 monoclonal antibody) in rheumatoid arthritis patients during a phase II clinical trial. J Clin Pharmacol 45:792-801

51. Li J, Levi M, Charoin J-E et al (2007) Rituximab exhibits a long half-life based on a population pharmacokinetic analysis in nonHodgkin's lymphoma (NHL) patients. Blood 110:700 [Abstract 2371]

52. Darwish M, Burke JM, Hellriegel E et al (2014) An evaluation of the potential for drug-drug interactions between bendamustine and rituximab in indolent non-Hodgkin lymphoma and mantle cell lymphoma. Cancer Chemother Pharmacol 73:1119-1127. doi:10.1007/s00280-014-2445-5

53. Regazzi MB, Iacona I, Avanzini MA et al (2005) Pharmacokinetic behavior of rituximab: a study of different schedules of administration for heterogeneous clinical settings. Ther Drug Monit 27:785-792

54. Berinstein NL, Grillo-López AJ, White CA et al (1998) Association of serum rituximab (IDEC-C2B8) concentration and antitumor response in the treatment of recurrent low-grade or follicular non-Hodgkin's lymphoma. Ann Oncol 9:995-1001

55. Iacona I, Lazzarino M, Avanzini MA et al (2000) Rituximab (IDEC-C2B8): validation of a sensitive enzyme-linked immunoassay applied to a clinical pharmacokinetic study. Ther Drug Monit 22:295-301

56. Lazzarino M, Arcaini L, Bernasconi P et al (2002) A sequence of immune-chemotherapy with rituximab, mobilization of in vivo purged stem cells, high-dose chemotherapy and autotransplant is an effective and non-toxic treatment for advanced follicular and mantle cell lymphoma. Br J Haematol 116:229-235 